AUTOMATIC NUMBER PLATE RECOGNITION USING CANNY EDGE

DETECTOR, SLIDING CONCENTRIC WINDOWS AND

\title{
LINEAR DISCRIMINANT ANALYSIS
}

\author{
BRINTHAKUMARI. $\mathbf{S}^{\mathbf{1}} \&$ S. K. MUTHUSUNDAR ${ }^{2}$ \\ ${ }^{1}$ Research Scholar, Computer Science and Engineering, Meenakshi Academy of Higher Education \\ and Research University, Tamil Nadu, India \\ ${ }^{2}$ Professor, Computer Science and Engineering, Sri Muthukumaran Institute of Technology,
}

Chennai, Tamil Nadu, India

\begin{abstract}
This paper presents a novel methodology of detection and recognizing characters in number plates in vehicles using Canny Edge Detector, Sliding Concentric Windows and Linear Discriminant Analysis. This framework of number plate recognition plays a vital role in parking places, toll gates, traffic monitoring, security control etc. This framework is implemented as a four step process. In the first step, the accepted image is preprocessed using Canny Edge Detector. This is a multi-stage algorithm that extracts wide variety of edges even in a noisy environment. The second step follows Sliding Concentric Windows (SCWs) for plate detection. License plate is extracted based on the vertical and horizontal position histogram and then morphological operations such as dilation and erosion are applied to get the right candidate plate region. The third step is character segmentation using Connected Component Analysis (CCA). Final step is for character recognition and it uses Linear Discriminant Analysis (LDA) using Local Binary Pattern (LBP) features. This work is implemented on the UFPR-ALPR Dataset and the experimental results shows the recognition accuracy of $88.5 \%$.

KEYWORDS: Canny Edge Detector, Sliding Concentric Windows, Linear Discriminant Analysis \& Local Binary Pattern Features
\end{abstract}

Received: Sep 04, 2018; Accepted: Sep 24, 2018; Published: Oct 27, 2018; Paper Id.: IJCNWMCDEC20181

\section{INTRODUCTION}

Automatic License Plate Recognition helps to identify vehicle in an efficient manner and with good recognition accuracy, without the need for major human resources and has become more and more important the recent years. There are several reasons for the importance of recognition of number plates in the vehicles. There are a growing number of vehicles on the roads with fast moving rate that cannot be tracked efficiently. The rapid development in image processing and computer vision technology has also made it possible to detect, identify and recognize the numbers in the license plates at a fast rate.

License Plate Recognition systems have been implemented and automated strictly in many countries throughout the world. The proposed work extracts the features like height and width of the plate, shape of the plate, color and font of the characters embedded in the plate. Even if there exist standard pattern of number plates in India, people follow their own pattern of styles. The variations are shown in terms of fonts, letter styles, font size, position of characters, color of characters and number plates. 
The ultimate aim of this work is to design a deployable working system for Indian License Plate Recognition which is a challenging task since there is no uniformity in the Indian License Plates, as each state has adopted its own style and structure. In addition to the problems faced by the General License Plate Recognition Algorithms, there are some set of specific problems needs to be solved by the Indian License Plate Recognition algorithms.

As there is inadequacy in the Indian Transportation Engineering and Infrastructure, the road rules are violated and by the lack of monitoring capability, the number of accidents gets increased. The primary need is to identify the vehicles using their license plates. But there prevails a non-uniformity in the Indian License plates and infrastructure.

The License plates may not be positioned in the correct position, according to the class of vehicles. This will lead to the misclassification of plate image in the vehicle. Number plates of other countries are also misclassified. In highway toll collection booths, the vehicles are queuing up for long hours and it becomes worst in peak hours to get the fee paid and pass on the gates. By using license plate recognition systems, automatically the fee can be collected from the owners account and no manual process in required in toll gates. The accidents are getting increased due to speed violation and disobeying the traffic rules. These kinds of violations can easily be monitored by this system and such driving can be penalized. Hence, if such systems are implemented, the best value from the road and rail systems can be prevailed and it also helps in traffic flow more smoothly, reducing delays, fuel consumption, and air and noise pollution.

The proposed work is purely based on Indian License plates and hence an overview of the same is very much essential. In India, by law, all license plates also known as number plate must be based on Hindu-Arabic numerals with Roman alphabets. Being a multi-linguistic country, in many states the number plates will have local scripting too and hence the Indian license plates are unique from other countries.

This paper is organized as follows. Related work is described in Section 2. Section 3 presents the data and methods. Section 4 presents proposed work describing the process to detect the characters in the number plates of the vehicles with good accuracy. Sections 5 presents the experimental results and Section 6 concludes the paper with some recommendations for future work.

\section{RELATED WORK}

Several techniques exist for automatic detection of license plate.

In [1] Sarfraz. M Ahmed. M. J et al presents Automatic License Plate Recognition systems (LPR) which helps to decrease the number of traffic violations and construct the streets safer. In that scheme they explained about an urbanized automatic scheme that locates Saudi license plates in a captured image regardless of the time and the license plate scale. The proposed system can stand slight tilting of the license plate. The localization process is complex due to the extreme varying nature of the background content. Good results were obtained using the localization stage. A second division of the system was developed for segmenting and distinguishing the characters in the positioned license plate. Locating the license plate in a picture is the first velocity in any license plate recognition system.

In [2] Suri. Dr. P. K, VermaEr. A et al the presentation is about the numbers on vehicle plates which is used as the input image and simple color conversion are used. Edge detection and connector measurement methods are also accounted too. Throughout the whole work, they use the masking and smoothing operations. The median filter is worn as one of the operators. The best consequences can be obtained by receiving the value of connector mechanism which is more than 17 . Various methods have been planned so far but there is always a difficulty to notice the numbers. The image is stored in the 
form of a matrix and the output is displayed in the shape of detected numbers. They use Sobel Edge Detection technique to minimize the complexity (as in covariance matrix) and local variance scores have been used and the sole coefficients have been efficient into a attribute vector form and multi-layer neural network. Since no explicit comparison or detachment calculation is essential in this framework, it is very easily possible to maintain the computational weight of the detection process low always.

S. Wang and H. Lee [3] combined edge statistics with morphological steps to eliminate unwanted edges in the processed images. On the other hand, some approaches take advantage of color features. Lin Luo [4] creates a new and effective approach of license plate location. The following three key sections are involved in this proposed algorithm. Firstly, Sobel operator is used to extract the vertical edges of the vehicle image. Then, HSV color space and integral image are employed to locate candidates in yellow license plates and non-yellow license plates. Finally connected component analysis is used to locate the license plate accurately.

Similarly, Yao-quan [5] also uses a color-based method, but in which the color information from the colored image is reasonably utilized to greatly decrease the edge points. With this method, it also eliminates the disturbances of the fake plate's region whose structure and texture are similar to the vehicle plate but do not match the plate's fixed color collocation.

The large contrast between the characters and the background is exploited in [6] to detect license plates with black characters over white backgrounds. While some other algorithms assumed that the density of edges in the license plate region is larger than other regions if the contrast of the character and the license plate is sufficiently large. For example, in $[7,8]$ they scanned the vehicle images with N-row distance to count the existent edges. Regions with high edge density will likely have the license plate inside. Similarly, in [9], a block-based method was proposed, and blocks with high edge magnitude and variance are considered as the license plate region.

Image transformation methods based on Hough transform, Gabor filters and wavelet transform have been applied in license plate detection. Hough transform is a classic algorithm to detect straight lines. Since the shape of license plate can be defined by lines, [10] used the Hough transform to detect the boundary of a license plate. This method is practical only when the background of the image is simple. Another disadvantage of this method is that the computational complexity of Hough transform is very high. Gabor filters are often used to analyze textures as they are sensitive to textures with different directions and scales, data and methods.

\section{DATASET - MEDIA LAB LPR DATABASE}

This work is implemented on the benchmark dataset, English LP Database. This database contains 530 images of $640 \times 480$ resolution with various illuminations. 


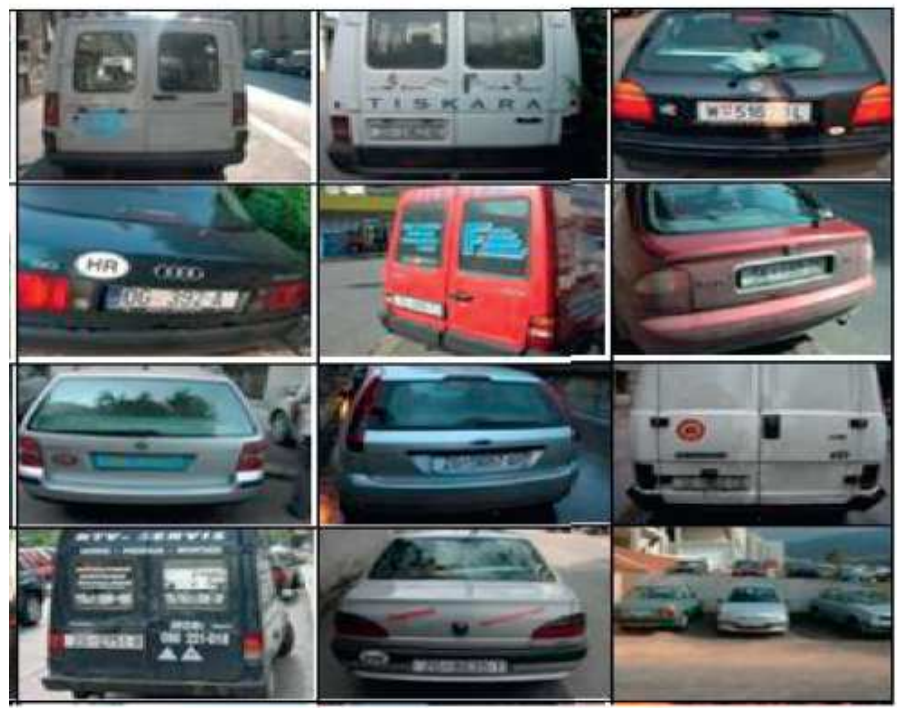

Figure 1: Some Vehicle Images from Original Dataset

The entire flow of the proposed work is depicted in figure 2. The implementation comprises of four main steps that includes (i) Preprocessing - Canny edge detector (ii) Plate detection using Sliding Concentric Windows (iii) Character Segmentation using Connected Component Analysis (iv) Character Recognition using Linear Discriminant Analysis of Local Binary Pattern features.

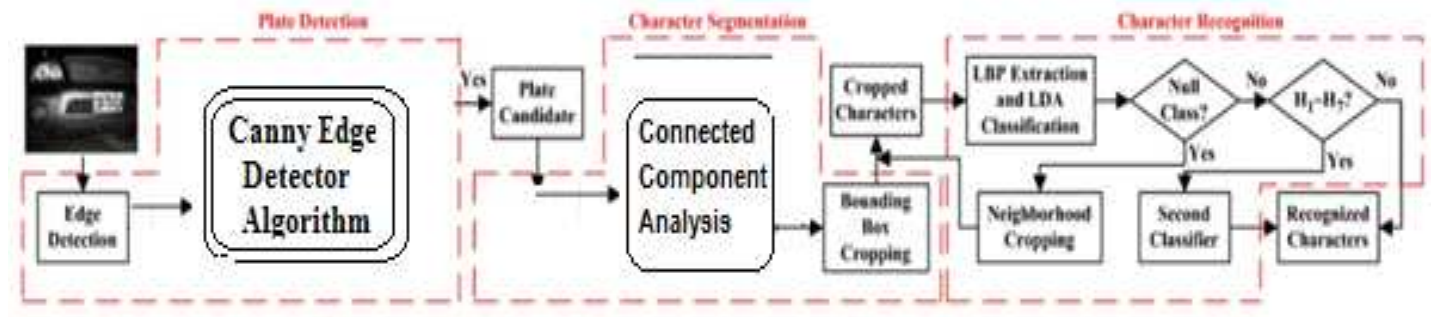

Figure 2: The Proposed Architecture of License Plate Recognition

\section{METHODOLOGY}

\section{Canny Edge Detector}

Edge in Vehicle Number plate provides very useful information for the recognition of number plate. Canny Edge Detector extracts edge information for several edges. The standardized number plates followed in our nation are with the following attributes: Seven characters in the plate, the size of each character is $90 \mathrm{~cm} \mathrm{X} 45 \mathrm{~cm}$, with $10 \mathrm{~cm}$ space between edge and character, $12 \mathrm{~cm}$ spacing between the characters.

The Canny Edge Detector algorithm extracts wide variety of edges even in noisy environment and the pseudo code is shown in figure 3 . 
1. Use the Gaussian filter $G \sigma(m, n)$ to smooth out the image $\mathrm{f}(\mathrm{m}, \mathrm{n})$.

2. Compute gradient of $g(m, n)$ using any of the gradient operators to reach $\mathrm{M}(\mathrm{m}, \mathrm{n})$

3. Threshold is given by $M_{T} m, n=0 \mid M(m, n)$

4. Suppress non-maxima pixels in the edges of MT are obtained above to thin the edge ridges. Check whether each non-zero MT $(m, n)$ is greater than its two neighbors along the gradient direction $\theta(\mathrm{m}, \mathrm{n})$. If so, keep MT $(\mathrm{m}, \mathrm{n})$ unchanged, otherwise, set it to zero.

5. Threshold the previous result by two different thresholds $\tau 1$ and $\tau 2$ (where $\tau 1<$ $\tau 2)$ to obtain two binary images $\mathrm{T} 1$ and $\mathrm{T} 2$. This $\mathrm{T} 2$ has less noise and fewer false edges than $\mathrm{T} 1$ but it also has more gaps between those edge segments.

6. Link edges segments in $\mathrm{T} 2$ to form continuous edges. To do so, trace each segment in $\mathrm{T} 2$ to its end and then search its neighbors in $\mathrm{T} 1$ to find any edge segment in T1 until it reaches another edge segment in T2.

Figure 3: Algorithm of Canny Edge Detector

\section{Sliding Concentric Windows Algorithm}

In the second step of License plate detection, sliding concentric windows method is used. The task of license plate detection is carried out in two steps. In the first step, a concentric window (A) is scanned from left to right and another concentric window (B) is scanned from top to bottom. Then features like mean and standard deviation of A and B are computed. Then evaluate the ratio between the two. If the ratio is greater than a threshold value, the central pixel of the window indicates the Region of Interest (ROI). It is indicated as 1 for ROI otherwise 0.

In the second step, extract the intensity component. From the intensity feature explore the green, yellow and white component values using Hue, Saturation and Intensity values.

Then generate the histogram for the license plate. And using the vertical and horizontal position of histogram generate the exact plate portion of the license plate.

Finally, Dilation and Erosion are applied to extract the right plate region.
1. Construct the image matrix, $A$
2. Construct the zero matrix, D
3. Define the structuring element, $B$
4. Pad matrix A with 0 on both sides
5. Place B on A and perform logical AND
6. If all values are zero update $D$ with 1
7. Repeat the above steps for all elements of A

Figure 4: Algorithm of Dilation

\section{Connected Component Analysis}

Connected Component Analysis, CCA, is one of the most widely selected algorithms for the initial step of character recognition in various segmentation methods. The connected component operator performs the scan from left to 
right till it reaches a point $\mathrm{p}$, for which the ROI is 1 . Then find the four neighbors of $\mathrm{p}$. Check whether if all the four neighbors are 0 , then assign a new label to $\mathrm{p}$. If only one neighbor is 0 , then assign it to $\mathrm{p}$. If more than one neighbors have 1 , then assign 1 as label of $\mathrm{p}$. Connected components labeling scans an image and groups its pixels into components based on pixel connectivity.

\section{Linear Discriminant Analysis}

Each character candidate obtained from the previous segmentation phase comes with an aspect ratio $r_{a}$ and orientation $\angle \mathrm{L}_{\mathrm{M}}$

If $r_{a}$ is not in the range $[1.6,2.5]$ or $\angle \mathrm{L}_{\mathrm{M}}$ is not within $\left[80^{\circ}, 100^{\circ}\right]$, showing that the character can be with a large viewpoint rotation, an affine transform would be applied to warp it to within the desired ranges of $r_{a}$ and $\angle L_{M}$.

The character candidate with appropriate aspect ratio and orientation is normalized to $30 \times 15$ pixels in scale and recognized by a hierarchical classifier with two processing layers. The first layer has 25 classes that cover all 35 alphanumeric characters 1 and one class that covers the non characters caused by plate edges, badly segmented characters or segments from cluttered backgrounds, giving a total of 26 classes. Out of the 25 alphanumeric classes, seven classes have an enhancement classifier in the second layer that targets a set of characters with high misclassification likelihoods. The seven sets are [B, R, 6, 8], [C, G], [D, 0], [E, F], [I, 1], [U, V], and [Z, 2, 7]. The remaining 18 classes in the first layer each identifies a specific character. The selection of the seven easy-to-misclassify sets is based on the classification results from the experiments that only use the first-layer classifier, which was originally designed to identify the 35 classes of alphanumeric characters.

\footnotetext{
1. Apply affine transformation

2. Normalize the image to $30 \times 15$ and partition into $10 \times 5$ cells

3. For each pixel $x$, compute its binary pattem $L$

4. Build histcgram for each cell as nine bines and obtain the feature $\mathrm{F}$ for I with 144 dimension.

5. Reduce the dimension using PCA and build LDA classifier for 26 classes in the first layer

6. Train LDA classifier for each character in the second layer
}

Figure 5: Algorithm of LDA Classifier

\section{EXPERIMENT EVALUATION AND RESULTS}

Using the benchmark dataset, English LP Database 200 images are taken as training samples and 150 images for testing and the rest for validation. The dataset is passed on the four modules sequentially one after the other. The work is implemented using MATLAB and tested in Windows PC with Pentium Dual Core 2.4-GHz processor.

The accuracy of Detection, Segmentation, Recognition of characters along with time taken for detection in Number Plate is specified in Table 1. 
Table 1: Accuracy of Character Detection in License Plate

\begin{tabular}{|c|c|c|c|c|c|c|}
\hline \multirow{2}{*}{ Letter } & \multirow{2}{*}{ Test Sample } & \multicolumn{4}{|c|}{ Accuracy (\%) } & \multirow{2}{*}{ Time(s) } \\
\cline { 3 - 6 } & & Detect & Segment & Recog & Overall & \\
\hline 0 & 210 & 90 & 89 & 85 & 88.2 & 0.06 \\
\hline 1 & 225 & 80 & 90 & 80 & 87.6 & 0.12 \\
\hline 2 & 300 & 78 & 89 & 78 & 78.6 & 0.10 \\
\hline 3 & 291 & 89 & 88 & 86 & 84.6 & 0.09 \\
\hline 4 & 223 & 79 & 88 & 75 & 80.2 & 0.12 \\
\hline 5 & 212 & 89 & 85 & 85 & 81.5 & 0.08 \\
\hline 6 & 235 & 88 & 80 & 86 & 81.6 & 0.09 \\
\hline 7 & 247 & 78 & 79 & 70 & 79.5 & 0.11 \\
\hline 8 & 301 & 84 & 86 & 92 & 87.4 & 0.13 \\
\hline 9 & 201 & 95 & 92 & 85 & 88.1 & 0.16 \\
\hline TN & 112 & 94 & 93 & 96 & 88.5 & 0.10 \\
\hline
\end{tabular}

The performance result shows the proposed work of detection of number plate outperforms other existing methods.

\section{CONCLUSIONS}

This work is implemented successfully with Canny Edge Detector for edge detection even in noisy environment, Sliding Concentric Windows (SCWs) with dilation and erosion to extract the number plate efficiently, Connected Component Analysis (CCA) for character segmentation and Linear Discriminant Analysis (LDA) using Local Binary Pattern (LBP) features for character recognition. This work is implemented on the English LP Database Dataset and the experimental results shows the recognition accuracy of $88.5 \%$.

In near future, other interest region detectors deserve to be studied for their potential in improving license plate recognition performance. The next version of the proposed work can include video samples of vehicle number plate for recognition with good accuracy.

\section{REFERENCES}

1. M. Rasooli, S. Ghofrani and E. Fatemizadeh, "Farsi License Plate Detection based on Element Analysis and Characters Recognition”, International Journal of Signal Processing, Image Processing and Pattern Recognition, vol. 4, no. 1, (2011) March, pp. 65-80.

2. M. I. Khalil, "Car Plate Recognition Using the Template Matching Method”, International Journal of Computer Theory and Engineering, vol. 2, no. 5, (2010) October, pp. 683-687.

3. S. Wang and H. Lee, "Detection and recognition of license plate characters with different appearances," IEEE Intelligent Transportation Systems, vol. 2, 2003.

4. L. Luo, H. Sun, W. Zhou and L. Luo, “An Efficient Method of License Plate Location” Information Science and Engineering (ICISE), 2009 1st International Conference on. Page: 770. 26-28 Dec. 2009.

5. Y. Yang, J. Bai, R. Tian, N. Liu, “ A vehicle license plate recognition system based on fixed color collocation,” Machine Learning and Cybernetics, 2005. Proceedings of 2005 International Conference on, Page: 5394, Issue Date: 18-21 Aug. 2005.

6. P. Comelli, P. Ferragina, M. N. Granieri, and F. Stabile, "Optical recognition of motor vehicle license plates," Vehicular Technology, IEEE Transactions on, vol. 44, pp. 790-799, 1995. 
7. Patel, A. M., Patel, A., \& Patel, H. R. (2013). Comparative Analysis For Machine Learning Techniques Appliance On Anomaly Based Intrusion Detection System For Wlan.

8. J. Cano and J.-C. Pérez-Cortés, Vehicle License plate segmentation in natural images vol. 2652. Berlin, ALLEMAGNE: Springer, 2003.

9. J. Barroso, E. L. Dagless, A. Rafael, and J. Bulas-Cruz, "Number plate reading using computer vision," in Industrial Electronics, 1997. ISIE '97., Proceedings of the IEEE International Symposium on, 1997, pp. 761-766 vol.3.

10. L. Hsi-Jian, C. Si-Yuan, and W. Shen-Zheng, "Extraction and recognition of license plates of motorcycles and vehicles on highways," in Pattern Recognition, 2004. ICPR 2004. Proceedings of the 17th International Conference on, 2004, pp. 356-359 Vol.4.

11. V. Kamat and S. Ganesan, "An efficient implementation of the Hough transform for detecting vehicle license plates using DSP'S," in Proceedings of the Real-Time Technology and Applications Symposium, 1995, p. 58. 